\title{
Quadratic Form Techniques and the Balslev-Combes Theorem
}

\author{
BARRY SIMON* \\ Departments of Mathematics and Physics, Princeton University, Princeton, N.J., USA
}

Received January 24, 1972

\begin{abstract}
We extend the theorem of Balslev and Combes on the absence of singular continuous spectrum to a class of interactions including $r^{-\alpha}(3 / 2 \leqq \alpha<2)$ local potentials. In addition, we note that the theory of sectorial operators allows a simplification of their proof and allows one to push the cuts through angles larger than the $\pi / 2$ restriction employed by Balslev-Combes.
\end{abstract}

\section{$\S 1$. Introduction}

In [1], Balslev and Combes introduced a powerful new technique to the mathematical theory of $N$-Body Schrödinger operators. This technique has already been used to prove the absence of singular continuous spectrum in the Hamiltonian of certain $N$-body systems [1] and to study time dependent perturbation theory $[11,12]$. Our main goal in this brief note is to show that the Balslev-Combes results can be extended to some other systems; in particular, to systems with central two body potentials, $V_{i j}(r)$, so that $V_{i j}$ has an analytic continuation to $\{r|| \arg r \mid<\alpha\}$ with $W_{\theta}(r)=V\left(e^{i \theta} r\right)$ in the Rollnik class [9], $R+\left(L^{\infty}\right)_{\varepsilon}$, if $|\operatorname{Im} \theta|<\alpha$. The result is a sufficiently straight forward merging of the techniques of [10] with those of [1] that Section 4 where we present the proof will be brief.

Our main goal in $[9,10]$ was to use quadratic form techniques to extend the theory of two body quantum mechanics to a large class of potentials. But we also found that form techniques could be used in simplifying the proofs and extending the theorems for the Kato classes. This is also true for the Balslev-Combes theory. Not only can we use form techniques to extend their theorem to a larger class $(\S 4)$ but we will show how notions from the theory of sectorial operators allow one to eliminate a difficult technical step from their induction ( $(3)$. In addition, we will show that the $|\operatorname{Im} \theta|<\pi / 4$ conditions that they place on their results are artifacts of the way they use Ichinose's lemma and that

$\star$ A. Sloan Foundation Fellow.

1 Commun math Phys., Vol 27 
the only limitation on $\operatorname{Im} \theta$ comes from the strip of analyticity for the potentials $(\$ 5)$.

It is a pleasure to thank Ed Nelson and Mike Reed for useful discussions; in particular Remark 3 following Theorem 1 is Nelson's.

\section{$\S 2$. The Class of Dilatation Analytic Form Interactions}

Combes [2] introduced the class, $C_{x}$, of dilatation analytic interactions. Let $H_{0}$ be $-\Delta$ on $L^{2}\left(\mathbb{R}^{3}\right)$ with the usual domain and let $\mathscr{H}_{+2}$ be the domain of $H_{0}, D\left(H_{0}\right)$, with the norm $\|\psi\|_{+2}^{2}=\left\|\left(H_{0}+1\right) \psi\right\|^{2}$ $=\left\langle\psi,\left(H_{0}+1\right)^{2} \psi\right\rangle$ and write $\mathscr{H}_{0}$ for $L^{2}\left(\mathbb{R}^{3}\right)$. Let $U(\theta)$ be the one parameter group of dilatations on $\mathscr{H}_{0}$ given by

$$
(U(\theta) f)(\boldsymbol{r})=e^{3 \theta / 2} f\left(e^{\theta} \boldsymbol{r}\right),
$$

$C_{x}$ was defined to be those mappings $V: \mathscr{H}_{+2} \rightarrow \mathscr{H}_{0}$ so that

(1) $V$ is $\mathscr{H}_{0}$-symmetric, i.e., $\langle\psi, V \psi\rangle$ is real for all $\psi \in \mathscr{H}_{+2}$.

(2) $V$ is compact as a map from $\mathscr{H}_{+2}$ to $\mathscr{H}_{0}$.

(3) The family of bounded operators $V(\theta)=U(\theta) V U(\theta)^{-1}$ from $\mathscr{H}_{+2}$ to $\mathscr{H}$ has an analytic continuation to a family of operators from $\mathscr{H}_{+2}$ to $\mathscr{H}$ into the strip $\{\theta|| \operatorname{Im} \theta \mid<\alpha\}$.

We introduce an analagous class $\mathscr{F}_{\alpha}$ defined in terms of forms. Let $\mathscr{H}_{+1}$ and $\mathscr{H}_{-1}$ be the scale spaces defined by $H_{0}\left[10, \S\right.$ II.2], so $\mathscr{H}_{+1}$ is the form domain of $H_{0}$ with norm

$$
\|\psi\|_{+1}^{2}=\left\langle\psi,\left(H_{0}+1\right) \psi\right\rangle
$$

and $\mathscr{H}_{-1}$ is the completion of $\mathscr{H}$ in the norm

$$
\|\psi\|_{-1}^{2}=\left\langle\psi,\left(H_{0}+1\right)^{-1} \psi\right\rangle,
$$

$\mathscr{H}_{+1}$ and $\mathscr{H}_{-1}$ are paired by a natural sesquilinear form $f: \mathscr{H}_{+1} \times \mathscr{H}_{-1} \rightarrow \mathbb{C}$ so that $f(\psi, \phi)=\langle\psi, \phi\rangle$ if $\phi \in \mathscr{H}_{0}$. We denote $f(\cdot, \cdot)$ by $\langle\cdot, \cdot\rangle$ henceforth.

Definition. $\mathscr{F}_{\alpha}$ is the class of all linear maps $V: \mathscr{H}_{+1} \rightarrow \mathscr{H}_{-1}$ so that

(i) $V$ is $\mathscr{H}_{0}$-symmetric, i.e., $\langle\psi, V \psi\rangle$ is real for all $\psi \in \mathscr{H}_{+1}$.

(ii) $V$ is compact as a map of $\mathscr{H}_{+1}$ to $\mathscr{H}_{-1}$.

(iii) The family of bounded operators $V(\theta)=U(\theta) V U(\theta)^{-1}$ from $\mathscr{H}_{+1}$ to $\mathscr{H}_{-1}$ has an analytic continuation to a family of operators from $\mathscr{H}_{+1}$ to $\mathscr{H}_{-1}$ into the strip $\{\theta|| \operatorname{Im} \theta \mid<\alpha\}$.

We are familiar from [10] that classes of interactions defined by operator conditions are contained in their form analogues, e.g. $L^{2}+L^{\infty}$ $C R+L^{\infty}$. This is true here:

Theorem 1. For any $\alpha>0, C_{\alpha} \subset \mathscr{F}_{\alpha}$. 
Remarks. 1. $\mathscr{F}_{\alpha}$ is however not contained in $C_{\alpha}$. For example, if $V$ is a function analytic in the sector $\{z|| \arg z \mid<\alpha\}$ with the functions $V_{i \beta}$ on $\mathbb{R}^{3}$ given by $V_{i \beta}(\boldsymbol{r})=V\left(|\boldsymbol{r}| e^{i \beta}\right)$ in $R+L_{\varepsilon}^{x}$ if $|\beta|<\alpha$, then $V \equiv V_{i 0}$ is in $\mathscr{F}_{\alpha}$. In particular, $V=r^{-\gamma}$ is in $\mathscr{F}_{\alpha}$ if $0<\gamma<2$ and is in $C_{\alpha}$ only if $0<\gamma<3 / 2$.

2. The symmetric nature of $V$ is absolutely critical. The result is false if we remove that requirement.

3. One can see why information from $\mathscr{H}_{+2} \rightarrow \mathscr{H}_{0}$ and $\mathscr{H}_{0}$-symmetry imply information about $\mathscr{H}_{+1} \rightarrow \mathscr{H}_{-1}$ by the language of interpolation; for if $V$ is $\mathscr{H}_{0}$ symmetric, information about $V: \mathscr{H}_{+2} \rightarrow \mathscr{H}_{0}$ implies information about $V: \mathscr{H}_{0} \rightarrow \mathscr{H}_{-2}$. "Interpolation" then gives information from $\mathscr{H}_{+1} \rightarrow \mathscr{H}_{-1}$.

Lemma 1. If $V$ is $\mathscr{H}_{0}$-symmetric and bounded from $\mathscr{H}_{+2}$ to $\mathscr{H}_{0}$, then it is bounded from $\mathscr{H}_{+1}$ to $\mathscr{H}_{-1}$ and $\|V\|_{+1,-1} \leqq\|V\|_{+2,0}$.

Proof. This is a standard fact ([5] or [3; Theorem V. 4.12]) but we give a proof due to Nelson which we need in Lemma 2. Translated into information about operators from $\mathscr{H}_{0}$ to $\mathscr{H}_{0}$, we need show that if $V\left(H_{0}+1\right)^{-1}$ is bounded, then $\left(H_{0}+1\right)^{-1 / 2} V\left(H_{0}+1\right)^{-1 / 2}$ (defined initially only on $\mathscr{H}_{+1}$ ) is bounded and

$$
\left\|\left(H_{0}+1\right)^{-1 / 2} V\left(H_{0}+1\right)^{-1 / 2}\right\| \leqq\left\|V\left(H_{0}+1\right)^{-1}\right\| .
$$

Since $V$ is symmetric, for any $\psi \in \mathscr{H}_{+2}$

$$
\left\|\left[\left(H_{0}+1\right)^{-1} V\right] \psi\right\|=\left\|\left[V\left(H_{0}+1\right)^{-1}\right]^{*} \psi\right\| \leqq\left\|V\left(H_{0}+1\right)^{-1}\right\|\|\psi\|
$$

so $\left(H_{0}+1\right)^{-1} V$ defined on $\mathscr{H}_{+2}$ extends to a bounded operator on $\mathscr{H}$ and $\left\|\left(H_{0}+1\right)^{-1} V\right\|=\left\|V\left(H_{0}+1\right)^{-1}\right\|$. Now let $\psi \in C^{\infty}\left(H_{0}\right)$ and let $f(z)$ $=\left(H_{0}+1\right)^{-z} V\left(H_{0}+1\right)^{z-1} \psi$. Then $f(z)$ is analytic and uniformly bounded in the strip $0 \leqq \operatorname{Re} z \leqq 1$. Thus by the Hadamard three line theorem

$$
\begin{aligned}
\left\|f\left(\frac{1}{2}\right)\right\| & \leqq \sup _{\operatorname{Re} z=0 \text { or } \operatorname{Re} z=1}\|f(z)\| \\
& \leqq \max \left(\left\|V\left(H_{0}+1\right)^{-1}\right\|\|\psi\|,\left\|\left(H_{0}+1\right)^{-1} V\right\|\|\psi\|\right) .
\end{aligned}
$$

Thus for $\psi \in C^{\infty}\left(H_{0}\right)$,

$$
\left\|\left(H_{0}+1\right)^{-1 / 2} V\left(H_{0}+1\right)^{-1 / 2} \psi\right\| \leqq\left\|V\left(H_{0}+1\right)^{-1}\right\|\|\psi\|
$$

which proves the result.

Lemma 2. If $V$ is $\mathscr{H}_{0}$ symmetric and compact as an operator from $\mathscr{H}_{+2}$ to $\mathscr{H}_{0}$, it is compact as an operator from $\mathscr{H}_{+1}$ to $\mathscr{H}_{-1}$.

Proof, We are given that $V\left(H_{0}+1\right)^{-1}$ is bounded so as in the proof of Lemma $1,\left(H_{0}+1\right)^{-\alpha} V\left(H_{0}+1\right)^{\alpha-1}$ is bounded if $0 \leqq \alpha \leqq 1$. Let $A(z)$ $=\left(H_{0}+1\right)^{-z} V\left(H_{0}+1\right)^{z-1}$. Then $A(z)$ is analytic in $R=\{z \mid 0<\operatorname{Re} z<1\}$ 
and continuous in $\bar{R}$. Moreover, $A(z)$ is assumed compact if $\operatorname{Re} z=0$. It follows that $A(z)$ is compact for all $z$ (see e.g. [8]). ${ }^{1}$

Proof of Theorem 1. Let $V \in C_{\alpha}$. Then $V$ defines a bounded map of $\mathscr{H}_{+1}$ to $\mathscr{H}_{-1}$ by Lemma 1 and $\langle\psi, V \psi\rangle$ is real for $\psi \in \mathscr{H}_{+1}$ since it is real for $\psi \in \mathscr{H}_{+2}$ which is $\|\cdot\|_{+1}$-dense in $\mathscr{H}_{+1}$. Thus (i) is true. By Lemma 2, conditions (1) and (2) for $V \in C_{\alpha}$ imply condition (ii) for $V$. Finally, note that since $\langle\psi, V(\theta) \psi\rangle$ is real when $\theta$ is real, $V(\bar{\theta})+V(\theta)$ and $i(V(\theta)-V(\bar{\theta}))$ are $\mathscr{H}_{0}$-symmetric, so by Lemma $1, V(\theta)$ is a bounded map from $\mathscr{H}_{+1}$ to $\mathscr{H}_{-1}$ for any $\theta$ with $|\operatorname{Im} \theta|<\alpha . V(\theta)$ is analytic as maps from $\mathscr{H}_{+1}$ to $\mathscr{H}_{-1}$ since the $V(\theta)$ are uniformly bounded on compacts (Lemma 1 again) and $\langle\psi, V(\theta) \phi\rangle$ is analytic for all $\phi \in \mathscr{H}_{+2}, \psi \in \mathscr{H}$.

\section{§3. Ichinose's Lemma and Sectoriality}

The results of Balslev and Combes are essentially corollaries of one main theorem on the spectrum of certain of certain nonself-adjoint operators $H(\theta)$ (see Theorem 3 below). Their proof depends on the application of the following lemma of Ichinose [4]:

Ichinose's Lemma. Let $A_{1}$ and $A_{2}$ be closed, densely defined operators in the separable Hilbert spaces $\mathscr{H}_{1}, \mathscr{H}_{2}$ obeying

$(\alpha) \sigma\left(A_{i}\right) \subseteq\left\{z|| \arg z \mid \leqq \frac{1}{2} \phi_{i}\right\} ; \phi_{1}+\phi_{2}<2 \pi$,

(B) $\left\|\left(A_{i}-\varrho e^{i \phi}\right)^{-1}\right\| \leqq C_{i}(\phi) \varrho^{-1}$ for $|\phi|>\frac{1}{2} \phi_{i}$.

Then $A=A_{1} \otimes I+I \otimes A_{2}$ defined on finite sums of vectors of the form $\psi_{1} \otimes \psi_{2} ; \psi_{i} \in D\left(A_{i}\right)$ is closable and its closure $\tilde{A}$ obeys

$$
\sigma(\tilde{A})=\sigma\left(A_{1}\right)+\sigma\left(A_{2}\right) .
$$

The proof of the main Balslev-Combes theorem is inductive; the harder part of the proof is to verify $(\beta)$ at each point in an inductive argument. We want to show that the applicability of Ichinose's lemma (and in particular, $(\beta)$ ) follows directly from "sectoriality" considerations.

Given $z_{0} \in \mathbb{C}, \beta \in[0,2 \pi]$ and $\phi \in[0, \pi]$, we define the sector

$$
S_{z_{0}, \beta}(\phi)=\left\{z|| \arg \left[e^{-i \beta}\left(z-z_{0}\right)\right] \mid \leqq \phi / 2\right\}
$$

$\phi$ is called the opening angle of $S, z_{0}$ and $\beta$ the vertex and direction of $S$.

Recall [6] that a quadratic form $a$ on a form domain $Q(a)$ is called strictly sectorial if $N(a) \equiv\{a(\psi, \psi) \mid \psi \in Q(a) ;\|\psi\|=1\}$ is contained in some sector of opening angle $\phi<\pi$. By varying $z_{0}$ and $\theta$ we can often find sectors of arbitrarily small opening angle containing $N(a)$. For example if $N(a)=\{z \mid \operatorname{Re} z \geqq 0,0 \leqq \operatorname{Im} z \leqq 1\}, N$ is not contained in a sector of opening angle 0 but by taking $z_{0}$ very negative and $\theta=0$ we can choose $\phi$ arbitrarily small.

\footnotetext{
1 See Note added in proof.
} 
Definition. Let $a$ be a quadratic form. We say $a$ is a form with arbitrarily small opening angle, if for any $\phi>0$, there is a $z_{0}$ and $\beta$ with

$$
N(a) \subset S_{z_{0}, \beta}(\phi) \text {. }
$$

We note first the elementary:

Proposition 1. Let $b$ be a quadratic form with arbitrary small opening angle. Suppose $a$ is a tiny form perturbation of $b$, i.e., $Q(a) \supset Q(b)$ and for any $\alpha>0$, there is a $\beta$ with

$$
|a(\psi, \psi)| \leqq \alpha|b(\psi, \psi)|+\beta\|\psi\|^{2}
$$

for all $\psi \in Q(b)$. Then $b+$ a defined on $Q(b)$ is a form with arbitrarily small opening angle.

We also recall that $a$ is called closed if $Q(a)$ with the norm $\|\psi\|_{+1}^{2}$ $=\operatorname{Re}\left(e^{-i \beta} a(\psi, \psi)\right)+\left(\left|z_{0}\right|+1\right)\|\psi\|^{2}$ is a Banach space and that $[6$; Chapter VIII and $\mathrm{X}]$; see also [3]:

Proposition 2. Let a be a closed sectorial quadratic form. Then there is a closed operator, A, obeying

(i) $D(A)$ is dense in $D(a)$ in $\|\cdot\|_{+1}$,

(ii) $a(\psi, \psi)=(\psi, A \psi)$ for all $\psi \in D(A)$,

(iii) If $S$ is a sector for a, then $\sigma(A) \subset S$ and if $z \notin S,\left\|(A-z)^{-1}\right\|$ $\leqq \operatorname{dist}(z, S)^{-1}$.

Moreover, $A$ is uniquely determined by (i), (ii), the condition that some $z \notin S$ be in the resolvent set for $A$, and the condition that $A$ be closed.

Proposition 3. Any sectorial form coming from an operator with a sector of opening angle $\phi<\pi$ is closable, i.e., has a smallest closed extension.

These facts and Ichinose's lemma imply a theorem about tensor products of sectorial operators (see also [7]):

Proposition 4. Let $a_{1}$ and $a_{2}$ be closed sectorial forms on separable Hilbert spaces $\mathscr{H}_{1}$ and $\mathscr{H}_{2}$ and let $A_{1}$ and $A_{2}$ be the associated operators. Suppose $a_{i}$ has a sector with opening angle $\phi_{i}$ and $\phi_{1}+\phi_{2}<\pi$. Let $D(a)$ be the finite linear combinations of $\psi_{1} \otimes \psi_{2}$ with $\psi_{i} \in Q\left(a_{i}\right)$ and define a by

$$
a\left(\psi_{1} \otimes \psi_{2}, \psi_{1} \otimes \psi_{2}\right)=\left[a,\left(\psi_{1}, \psi_{1}\right)\right]\left(\psi_{2}, \psi_{2}\right)+\left(\psi_{1}, \psi_{1}\right)\left[a_{2}\left(\psi_{2}, \psi_{2}\right)\right]
$$
Then:

(a) a is a sectorial form,

(b) if $A$ is the operator associated with the closure of a, then $\sigma(A)$ $=\sigma\left(A_{1}\right)+\sigma_{2}\left(A_{2}\right)$. 
Proof. (a) Follows from the fact that $\phi_{1}+\phi_{2}<\pi$. By Proposition 2, Ichinose's lemma is applicable so we need only show $A=\tilde{A}$, the operator closure of $A_{1} \otimes 1+1 \otimes A_{2}$. But a simple argument shows that $D(\tilde{A})$ is dense in $D(a)$ in $\|\cdot\|_{+1}$ and clearly $\langle\psi, \tilde{A} \psi\rangle=a(\psi, \psi)$ for any $\psi \in D(\tilde{A})$. Finally, by Ichinose's lemma some $z \notin S$ is in the resolvent set for $\tilde{A}$ so $A=\tilde{A}$.

Throughout the remainder of this paper, we deal with Hamiltonians of $\mathrm{N}$-Body systems with their center of mass motion removed. We use coordinates $\boldsymbol{r}_{i}$ of the $i$ th particle relative to the $N$ th and write $\boldsymbol{r}_{i 0} \equiv \boldsymbol{r}_{i}$ and $\boldsymbol{r}_{i j}=\boldsymbol{r}_{i}-\boldsymbol{r}_{j}$. For each $i, j, L^{2}\left(\mathbb{R}^{3 N-3}\right)$ decomposes into $L^{2}\left(\mathbb{R}^{3}\right) \otimes L^{2}\left(\mathbb{R}^{3 N-6}\right)$ corresponding to the coordinate $r_{i,}$. If $v_{i j}$ is an operator on $L^{2}\left(\mathbb{R}^{3}\right)$, we write $V_{i j}=v_{i j} \otimes 1$ and write $V_{i j} \in \mathscr{F}_{\alpha}$ as shorthand for $v_{i j} \in \mathscr{F}_{\alpha}$. If each $V_{i j} \in \mathscr{F}_{\alpha}$ and $H=H_{0}+\sum_{i, j=0}^{N-1} V_{i j}$, we define

$$
H(\theta)=e^{-2 \theta} H_{0}+\sum_{i, j} V_{i j}(\theta) .
$$

Then Proposition 1 and the fact that $v_{i j}(\theta)$ is $h_{0}$ compact which implies that $v_{i j}$ is $K L M N$ small (so $V$ is KLMN small) tell us:

Theorem 2. If each $V_{i j} \in \mathscr{F}_{x}$ and $|\operatorname{Im} \theta|<\alpha$, then $H(\theta)$ is a sectorial form with arbitrarily small open angle.

As a result of Proposition 4 and Theorem 2, Ichinose's lemma is applicable without first proving directly that his condition $(\beta)$ holds by induction. This is a considerable simplification of the Balslev-Combes proof.

\section{$\S$ 4. Form Analogue of the Main Balslev-Combes Theorem}

In this section, we state and sketch the proof of the main BalslevCombes theorem [1] for the class $\mathscr{F}_{\alpha}$. By Theorem 1, this theorem is a strict extension of the basic theorem of [1].

Theorem 3. Let $V_{i j} \in \mathscr{F}_{\alpha} ; i, j=0, \ldots, n-1$. For $|\operatorname{Im} \theta|<\alpha$, let $H(\theta)$ $=e^{-2 \theta} H_{0}+\sum_{i, j} V_{i j}(\theta)$. For any partition $D=\left\{C_{1}, \ldots, C_{k}\right\}$ of $\{0, \ldots, N-1\}$ into disjoint sets, let $H_{C_{t}}(\theta)$ be the Hamiltonian for the particles in $C_{i}$ with center of mass removed (so if \# $\left(C_{i}\right)=0, H_{C_{t}} \equiv 0$ on $\mathbb{C}$ ). Let

$$
\sum_{\theta}=\bigcup_{D}\left\{E_{1}+\cdots+E_{k} \mid E_{\imath} \in \sigma_{\mathrm{disc}}\left(H_{C_{t}}(\theta)\right)\right\} .
$$

Then (a) $\sigma_{\mathrm{ess}}(H(\theta)) \subset\left\{\lambda+e^{-2 \theta} r \mid \lambda \in \sum_{\theta} ; r \geqq 0\right\}$.

(b) $\sum_{\theta}$ is only dependent on $\operatorname{Im} \theta$.

(c) If $\lambda \in \sum_{\theta_{0}}$, then $\lambda \in \sum_{\theta}$ for all $\theta$ sufficiently near $\theta_{0}$. 
Proof. 1. As in the Balslev-Combes paper, (b) and (c) follow from the Kato-Rellich perturbation theory and the fact that $H(\theta)$ and $H\left(\theta^{\prime}\right)$ are unitarily equivalent when $\operatorname{Im} \theta=\operatorname{Im} \theta^{\prime}$.

2. We prove (a) by induction on $N . N=1$ is trivial.

3. For fixed 0 , we make an analysis following [10] (§ VII.2).

In terms of "reduced disconnected parts", $D_{R}$ and "symmetrical connected interactions", $I_{s}$, one has a modified Weinberg-Van Winter equation ([10], egn. VII.16):

$$
(E-H(\theta))^{-1}=\left(E-H_{0}(\theta)\right)^{-1 / 2}\left(1-I_{\mathrm{s}}(\theta ; E)\right)^{-1} D_{R}(\theta ; E)\left(E-H_{0}(\theta)\right)^{-1 / 2} .
$$

Both $D_{R}(\theta ; E)$ and $I_{s}(\theta ; E)$ are finite sums of products of terms, each one, one of two types:

(i) $\left(E-H_{0}(\theta)\right)^{-1 / 2} V_{i J}\left(E-H_{0}(\theta)\right)^{-1 / 2}$; these are analytic if

$$
E \notin\left\{e^{-2 \theta} r \mid r \geqq 0\right\} .
$$

(ii) $\left(E-H_{0}(\theta)\right)^{1 / 2}\left(E-H_{D}(\theta)\right)^{-1}\left(E-H_{0}(\theta)\right)^{1 / 2}$ where $D$ has $k \geqq 2$ clusters. Each $H_{D}$ is of the form

$$
H_{C_{1}} \otimes \cdots \otimes 1+1 \otimes H_{C_{2}} \otimes \cdots \otimes 1+\cdots+1 \otimes \cdots \otimes H_{C_{k}} \otimes 1+1 \otimes \cdots \otimes H_{D}^{(0)}
$$

where $H_{D}^{(0)}$ is a relative kinetic energy. By the analysis in $\S 3$ and Ichinose's lemma, $\sigma\left(H_{D}\right)=\Sigma \sigma\left(H_{C_{1}}(\theta)\right)+\sigma\left(e^{-2 \theta} H_{0}\right)$. Since \# $C_{i}<N$ for all $i, \sigma\left(H_{D}\right) \subset\left\{\lambda+e^{-2 \theta} \mid \lambda \in \sum_{\theta} ; r \geqq 0\right\}$ by the induction hypothesis.

Thus $I_{s}(\theta ; E)$ and $D_{R}(\theta ; E)$ are analytic if $E \notin \sum_{\theta}+e^{-2 \theta} \mathbb{R}_{+}$.

4. Using a trick from $[8], I_{s}(\theta ; E)$ is compact for $E \notin \sum_{\theta}+e^{-2 \theta} \mathbb{R}_{+}$, for it is enough to prove it compact for $E e^{2 \theta}$ very negative (and use analytic continuation). But for $E e^{2 \theta}$ very negative, the perturbation series for $I_{s}(\theta ; E)$ converges in norm and is term by term compact.

5. By the "analytic Fredholm theorem", [10, p. 218; 6, Chapter VI] $(E-H(\theta))^{-1}$ has only discrete spectrum outside $\sum_{\theta}+e^{-2 \theta} \mathbb{R}_{+}$.

Theorem 3 has one critical consequence, proven in the exact same way as in [1]:

Theorem 4. Under the hypotheses of Theorem 3, $H$ has no singular continuous spectrum.

\section{§ 5. On the Balslev-Combes Condition, $|\operatorname{Im} \theta|<\pi / 4$}

In their paper, Balslev and Combes have a condition that $\alpha<\pi / 4$ for their analogue of Theorem 3 . This results from their applying Ichinose's lemma only to sectors $S_{z_{0}, \beta}(\phi)$ with $\beta=0$ and $z_{0}$ real. By taking $z_{0}$ and 
$\beta$ suitably, we have seen (Theorem 2) that Ichinose's lemma is applicable no matter what the value of $\theta$; the only limit on $\operatorname{Im} \theta$ is that imposed by the potentials (so, e.g. $\alpha=\pi / 2$ for Yukawa potentials). Of course, at $\theta=\pi / 2$, "the cuts" hit one another and this limits which sheets can be reached by continuation in $\theta$.

A particularly amusing case occurs when one deals with atoms; there, $V(\theta)=e^{-\theta} V$ so:

Theorem 5. In the case of atomic (i.e., Coulombic) Hamiltonians, $H(\theta)$ is an entire analytic family of type $(A)$, periodic with period $2 \pi i$. $H(\pi i)$ describes a self-adjoint system [namely $\left.H_{0}-V\right]$ in which all electronelectron forces are attractive and all nuclear-electron forces are repulsive.

\section{§ 6. Absence of Positive Energy Bound States in Atomic Systems}

The extension of the last section to arbitrary $\operatorname{Im} \theta$ allows us to recover Weidmann's result $[13,14]$ that purely Coulombic Hamiltonians have no positive energy bound states; in addition we obtain a result about resonances.

Theorem 6. Let $H$ be the Hamiltonian of a N-body system with $V_{i j}\left(r_{i j}\right)=C_{i j} /\left|\boldsymbol{r}_{i j}\right|$. Then $H$ has no bound states of energy $E>0$ and no positive energy resonances, in the sense that for $\theta$ with $0<\operatorname{Im} \theta<\pi / 2$, $H(\theta)$ has no eigenvalues in $\{\lambda \mid-2 \operatorname{Im} \theta<\arg \lambda<0\}$.

Proof. We use induction on $N . N=1$ is trivial. By induction $H(\theta)$ has no thresholds in $\{\lambda \mid-2 \operatorname{Im} \theta<\arg \lambda \leqq 0\}$. If $H(\theta)$ had an eigenvalue in the open sector, $H(\pi i)$ would have a complex eigenvalue since the eigenvalue avoids essential spectrum as $\theta \rightarrow i \pi$. Since $H(\pi i)=H_{0}-V$ is self-adjoint this is impossible. Now suppose $E_{0}>0$ is an eigenvalue of multiplicity $m$. By induction, $E=0$ is the largest threshold, so $E_{0}$ remains away from essential spectrum if $|\operatorname{Im} \theta|<\pi$. Thus $\psi_{1}(\theta), \ldots, \psi_{m}(\theta)$ are analytic in $|\operatorname{Im} \theta|<\pi$. Since $H( \pm \pi i)$ is also self-adjoint, $E_{0}$ remains an eigenvalue of multiplicity $m$ for $H( \pm \pi i)$ (see [1]). Thus $\psi_{i}(\theta)$ are entire functions of $\theta$. Since $H(2 \pi i)=H(0)$, there is a matrix $A$ on $\mathbb{C}^{m}$ with $\psi_{i}(2 \pi i)=\sum_{j=1}^{m} a_{i j} \psi_{j}(0)$. Choose $\phi=\Sigma C_{i} \psi_{j}(0)$ so that $A \phi=\alpha \phi$ for some $\alpha$. Letting $D$ be the infinitesimal generator of dilatations, we see that $\phi$ is in the domain of the unbounded self-adjoint operator $e^{2 \pi D}$ and $e^{2 \pi D} \phi$ $=\alpha \phi$. Thus $D \phi=\frac{1}{2 \pi}(\ln \alpha) \phi$. But $e^{i \theta D}$ goes weakly to 0 as $\theta \rightarrow \infty$, so $D$ has no eigenvalues. This contradiction proves that $H$ has no positive eigenvalues. 
Note added in proof. R. Solovay has pointed out a gap in the proof of Lemma 2, for all that is a priori clear is that $A(z)$ is strongly continuous in $\bar{R}$ (i.e. at the boundary $R$ ) while the argument of [8] requires norm continuity. The following lemma (which abstracts a suggestion of Solovay) fills the gap:

Lemma. Let $A(z)$ be a uniformly bounded weakly continuous operator valued function in $\bar{R}=\{z \mid 0 \leqq \operatorname{Re} z \leqq 1\}$, analytic in $R=\{z \mid 0<\operatorname{Re} z<1\}$. Suppose $A(z)$ is compact and norm continuous if $\operatorname{Re} z=0,1$. Then $A(z)$ is norm continous and compact in all of $\bar{R}$.

Proof. Since $A(z)=\|\cdot\|-\lim _{x \downarrow 0} e^{\alpha z^{2}} A(z)$ uniformly on compacts, it is sufficient to prove the theorem under the hypothesis $\lim _{z \rightarrow \infty}\|A(z)\|=0$. Under that hypothesis, let $A_{n}(z)=P_{n} A(z) P_{n}$ where $P_{n}$ is a family of finite rank projections with $s-\lim P_{n}=1$. Then each $A_{n}(z)$ is norm continuous and compact. When $\operatorname{Re} z=0,1, A_{n}(z) \rightarrow A(z)$ in norm uniformly on compacts, since $A(z)$ is then compact. Since $\|A(z)\| \rightarrow 0$ as $z \rightarrow \infty, A_{n}(z) \stackrel{\|\|}{\longrightarrow} A(z)$ uniformly for all $z$ with $\operatorname{Re} z=0,1$. By the maximum principle it follows that $A_{n}(z) \stackrel{H}{\longrightarrow} A(z)$ for all $z$ in the strip.

Remarks. 1. The proof can be modified to conclude compactness and norm continuity in $\{z \mid 0 \leqq \operatorname{Re} z<1\}$ if $A(z)$ is only assumed compact and norm continuous when $\operatorname{Re} z=0$.

2. To apply this lemma to the proof of Lemma 2, one must prove that $A(z)$ is norm continuous if $\operatorname{Re} z=0,1$. This follows from the fact that the product of a strongly convergent sequence and a fixed compact operator is norm convergent.

It is a pleasure to thank R. Solovay for pointing this error out to me.

\section{References}

1. Balslev, E., Combes, J. M.: Commun. math. Phys. 22, 280-294 (1971).

2. Combes, J.M.: CNRS technical report (unpublished).

3. Kato, T.: Perturbation theory for linear operators. Berlin-Heidelberg-New York: Springer 1966.

4. Ichinose, T.: Operational calculus for tensor products of linear operators in Banach spaces (to appear).

5. Löwner, K.: Math. Z. 38, 177 (1934).

6. Reed, M., Simon, B.: Methods of modern mathematical physics. I. New York: Academic Press (1972).

7. - - In preparation; see also A spectral mapping theorem for tensor products of unbounded operators. Bull. Amer. Math. Soc. (to appear).

8. Simon, B.: Helv. Phys. Acta 43, 607-630 (1970).

9. - Commun. math. Phys. 22, 269 (1971).

10. - Quantum mechanics for Hamiltonians defined as quadratic forms. Princeton: Princeton Univ. Press 1971.

11. - Phys. Letters A 36, 23 (1971).

12. - Resonances for dilatation analytic potentials and the foundations of time dependent perturbation theory (to appear).

13. Weidmann, J.: Commun. Pure Appl. Math. 19, 107 (1966).

14. - Bull. Amer. Math. Soc. 73, 452-456 (1967).

\section{B. Simon}

Departments of Mathematics and Physics

Princeton University

Princeton, New Jersey 08540, USA 